\title{
Significance of fragmented QRS complexes for identifying culprit lesions in patients with non-ST-elevation myocardial infarction: a single-center, retrospective analysis of 183 cases
}

\author{
Rong Guo, Yuanmin Li, Yawei Xu*, Kai Tang and Weimin Li
}

\begin{abstract}
Background: Fragmented QRS (fQRS) complexes are novel electrocardiographic signals, which reflect myocardial conduction delays in patients with coronary artery disease (CAD). The importance of fQRS complexes in identifying culprit vessels was evaluated in this retrospective study.

Methods: A 12-lead surface electrocardiogram was obtained in 183 patients who had non-ST-elevation myocardial infarction (NSTEMI) and subsequently underwent coronary angiography (CAG). On the basis of the frequency of fQRS complexes, indices such as sensitivity, specificity, positive and negative predictive values, and likelihood ratio were evaluated to determine the ability of fQRS complexes to identify the culprit vessels.

Results: Among the patients studied, elderly patients (age $\geq 65$ years) and those with diabetes had a significantly higher frequency of fQRS complexes ( $p=0.005, p=0.003$, respectively). The fQRS complexes recorded in the 4 precordial leads had the highest specificity (81.8\%) for indentifying the culprit vessel (left anterior descending artery). However, the specificity of fQRS complexes to identify lesions in the left circumflex and right coronary arteries was lower for the inferior and lateral leads than for the limb leads (65.5\% versus 71.7\%); however, the limb leads had higher sensitivity (92.3\% versus $89.4 \%)$. And the total sensitivity and specificity of fQRS $(77.1 \%$ and $71.5 \%)$ were higher than those values for ischemic T-waves.

Conclusions: The frequency of fQRS complexes was higher in elderly and diabetic patients with NSTEMI. The frequency of fQRS complexes recorded in each of the ECG leads can be used to identify culprit vessels in patients with NSTEMI.
\end{abstract}

Keywords: Fragmented QRS complexes, Coronary artery disease, Acute coronary syndrome, Electrocardiogram

\section{Background}

The diagnosis of non-ST-elevation myocardial infarction (NSTEMI) depends on a thorough physical examination, detailed clinical history, and immediate interpretation of the results of a resting 12-lead echocardiogram (ECG). Therefore, the ECG can provide crucial information for diagnosing NSTEMI. Other ECG abnormalities such as T-wave inversion, ST-segment depression, microvolt Twave alternans, late potentials on the signal-averaged

\footnotetext{
* Correspondence: xuyaweicn@yahoo.com.cn

Department of Cardiology, Shanghai Tenth People's Hospital, Tongji University School of Medicine, Shanghai 200072, China
}

ECG, and pathologic $\mathrm{Q}$ waves have diagnostic value, but their correlation with the exact anatomic location of the culprit lesion is not very high.

Fragmented QRS (fQRS) complexes are novel electrocardiographic signals, which reflect altered ventricular conduction delays around regions of a myocardial scar. The detection of $\mathrm{fQRS}$ complexes in a routine 12-lead ECG is a marker for abnormal cardiac depolarization. Previous studies have shown that $\mathrm{fQRS}$ complexes are useful for diagnosing coronary artery disease (CAD) $[1,2]$. However, limited information is available on the diagnostic utility of fQRS complexes in patients with NSTEMI. In this study, we reviewed the ECG results in 
NSTEMI patients to evaluate the accuracy of fQRS complexes to identify culprit lesions.

\section{Methods}

\section{Study population}

From January 2010 to April 2011, we conducted a retrospective study on 183 consecutive patients diagnosed with NSTEMI in our department. Standard 12-lead ECGs, data on cardiac biomarkers, and echocardiographic and coronary angiography (CAG) findings were collected from the study subjects. The patient demographics, including history of significant CAD (prior myocardial infarction or typical angina pectoris), previous percutaneous coronary intervention (PCI) or coronary artery bypass grafting (CABG), and risk factors for CAD (age, sex, hypertension, smoking, diabetes mellitus or hyperlipidemia) were noted.

Patients were eligible for inclusion in the study if they had a confirmed NSTEMI and an fQRS complex in any of the ECG leads recorded during the NSTEMI episode. Patients with permanent atrial fibrillation, ventricular paced rhythm, a previously implanted implantable cardioverter-defibrillator (ICD) or a clinical indication for an ICD at the time of enrollment, left ventricular hypertrophy, Wolff-Parkinson-White syndrome, cardiomyopathy, myocarditis, and congenital heart disease were excluded from the study. NSTEMI was defined as STsegment depression or prominent T-wave inversion and/ or positive tests for biomarkers of necrosis in the absence of ST-segment elevation detected in an appropriate clinical setting (i.e., when the patient experienced chest discomfort or angina) [3].

This study was approved by the institutional ethics committee, and informed consent was obtained from all enrolled patients.

\section{ECG}

The ECG and supplemental criteria for fQRS patterns were defined by Das [1,4]. The resting 12-lead ECG (filter range, $0.15-100 \mathrm{~Hz}$; AC filter, $60 \mathrm{~Hz}, 25 \mathrm{~mm} / \mathrm{s}$, $10 \mathrm{~mm} / \mathrm{mV}$ ) was analyzed by 2 independent readers blinded to the CAG results. The fQRS pattern was defined as the presence of an additional R' or crochetage wave, notching in the nadir of the $\mathrm{S}$ wave or fragmentation of the RS or QS complexes in 2 contiguous leads corresponding to a major coronary artery territory. The fQRS pattern could occur in patients with or without Q waves. However, patients with a typical bundle-branch block pattern $(\mathrm{QRS} \geq 120 \mathrm{~ms})$ or incomplete right bundle-branch block pattern were excluded. Furthermore, patients with a pathological Q wave in the ECG and a history of prior myocardial infarction were also excluded.

\section{CAG}

The severity of stenoses was graded using the Coronary Angiogram Analyzing System II (CAAS II; Pie Medical, Maastricht, The Netherlands)[5]. Coronary flow over the culprit lesion was graded according to the Thrombolysis in Myocardial Infarction Trial (TIMI) criteria, and collateral circulation was classified according to the criteria proposed by Rentrop et al [6,7]. Multivessel CAD was defined as the presence of lesions in 3 or more coronary vessels. The presence of occlusion in the main and secondary branch of a vessel was defined as single-vessel disease.

CAG was performed in all 183 patients included in the study. Of these patients, 87 underwent angiography during the first $24 \mathrm{~h}$ of the NSTEMI episode, and 96 underwent CAG within 2 to 14 days after the acute episode. The location of coronary lesions, the number of stenosed arteries and the degree of stenosis were recorded for each patient.

\section{Statistical analysis}

Values are expressed as mean \pm standard deviation. A two-tailed Student's $t$-test was used for comparing continuous variables. A Chi-square test or Fisher's exact test was used to compare dichotomous data. To evaluate the diagnostic value of fQRS complexes in each patient, indices such as sensitivity, specificity, likelihood ratio, and positive and negative predictive values were recorded. Logistic regression analysis was used to examine the influence of various clinical factors by estimating the probability of fQRS occurrence on an ECG. Receiver operating characteristic (ROC) curves were used to assess the relationship between $\mathrm{fQRS}$ complexes and ischemic T-waves for the diagnosis of NSTEMI. SPSS 13.0 was used for all statistical analyses. A $p$ value $<0.05$ was considered statistically significant.

\section{Results}

\section{Demographics and clinical data}

The ECG findings of the patients showed that fQRS complexes were present in $60.1 \%$ of all patients with NSTEMI $(n=183)$. The remaining 73 patients with ST-segment depression or prominent T-wave inversion formed the control group. Advanced age and the prevalence of diabetes were higher in the fQRS group $(p<0.05)$. No significant differences were observed in renal function between the two groups (Table 1). Logistic regression analysis showed that old age ( $\geq 65$ years), cardiac troponin $\mathrm{T}$ (cTnT) levels and diabetes were significantly associated with the presence of fQRS complexes. The odds ratios (OR) for old age, cTnT and diabetes were 2.04 (95\% CI, 1.09-3.09; $p=0.026$ ), 0.73 (95\% CI, 0.55$0.98 ; p=0.036)$ and 2.05 (95\% CI, 1.06-3.97; $p=0.033$ ), respectively (Table 2 ). 
Table 1 Baseline characteristics of enrolled patients

\begin{tabular}{llll}
\hline & $\begin{array}{l}\text { fQRS group } \\
(\mathbf{n}=\mathbf{1 1 0})\end{array}$ & $\begin{array}{l}\text { Non-fQRS group } \\
(\mathbf{n}=\mathbf{7 3})\end{array}$ & $\boldsymbol{p}$-value \\
\hline Age (yrs) & $64 \pm 1.0$ & $59 \pm 1.0$ & 0.005 \\
Gender (M/F) & $70 / 40$ & $47 / 26$ & 0.918 \\
Hypertension & $70.9 \%(78)$ & $63.0 \%(46)$ & 0.263 \\
Hyperlipidemia & $61.8 \%(68)$ & $67.1 \%(49)$ & 0.464 \\
Diabetes mellitus & $73.6 \%(81)$ & $52.1 \%(38)$ & 0.003 \\
Tobacco use & $58.2 \%(64)$ & $60.3 \%(44)$ & 0.778 \\
CTnT (ng/ml) & $1.35 \pm 0.12$ & $1.03 \pm 0.10$ & 0.049 \\
Prior PCl & $41.8 \%(46)$ & $46.6 \%(34)$ & 0.525 \\
Prior CABG & $11.8 \%(13)$ & $12.3 \%(9)$ & 0.917 \\
LVEF (\%) & $57.3 \pm 10.3$ & $57.1 \pm 11.1$ & 0.933 \\
Cockcroft-Gault formula & $81.16 \pm 15.67$ & $83.52 \pm 15.41$ & 0.299 \\
(CG-GFR) (ml/min) & & & \\
\hline
\end{tabular}

$\mathrm{M}=$ males; $\mathrm{F}=$ females; $\mathrm{cTnT}=$ cardiac Troponin $\mathrm{T} ; \mathrm{PCl}=$ percutaneous coronary intervention; $C A B G=$ coronary artery bypass grafting; $L V E F=$ left ventricular ejection fraction; Cockcroft-Gault formula (CG-GFR) was used to estimate renal function.

\section{CAG}

Out of the 183 patients, 42 showed left coronary artery dominance, 125 showed right dominance and 16 had a balanced coronary system. The incidence of triple-vessel disease was higher in the fQRS group than that in the control group $(p=0.002)$. The incidence of 3-vessels disease were quite higher in fQRS group $(\mathrm{p}=0.002)$. Similarly, severe and mild degree of coronary stenosis in fQRS group were much higher than that of non-fQRS group $(\mathrm{p}=0.038 ; \mathrm{p}=0.001)($ Table 3$)$.

\section{The diagnostic importance of fQRS complexes in the 12-lead ECG}

The frequency of $\mathrm{fQRS}$ recorded in each ECG lead was related to the culprit vessel or lesion in patients with

Table 2 Logistic regression analysis

\begin{tabular}{llll}
\hline & $\boldsymbol{p}$-value & OR & $\mathbf{9 5 \%} \mathbf{C l}$ \\
\hline Hypertension & 0.327 & 1.401 & $0.71-2.75$ \\
Hyperlipidemia & 0.824 & 0.928 & $0.48-1.80$ \\
Tobacco use & 0.565 & 1.212 & $0.63-2.33$ \\
Diabetes & 0.033 & 2.052 & $1.06-3.97$ \\
CTnT & 0.036 & 0.731 & $0.55-0.98$ \\
Prior PCl & 0.973 & 0.989 & $0.53-1.86$ \\
Prior CABG & 0.834 & 1.106 & $0.43-2.83$ \\
Gender & 0.632 & 0.936 & $0.48-1.83$ \\
LVEF & 0.909 & 0.998 & $0.97-1.03$ \\
Old age $(\geq 65$ yrs) & 0.026 & 2.042 & $1.09-3.84$ \\
\hline Valibles
\end{tabular}

Variable(s): hypertension, hyperlipidemia, tobacco use, diabetes, cardiac troponin $\mathrm{T}(\mathrm{cTnT})$, percutaneous coronary intervention $(\mathrm{PCl})$, coronary artery bypass grafting (CABG), gender, left ventricular ejection fraction (LVEF), old age.
Table 3 Comparison of CAG results between the 2 groups

\begin{tabular}{|c|c|c|c|}
\hline & $\begin{array}{l}\text { fQRS group } \\
(n=110)\end{array}$ & $\begin{array}{l}\text { Non-fQRS group } \\
(n=73)\end{array}$ & $p$-value \\
\hline \multicolumn{4}{|l|}{ Coronary Lesions } \\
\hline Single-vessel disease & 23 & 14 & 0.775 \\
\hline Double-vessel disease & 38 & 43 & 0.193 \\
\hline Triple-vessel disease & 49 & 16 & 0.002 \\
\hline \multicolumn{4}{|l|}{ Culprit vessel } \\
\hline LAD & 102 & 63 & 0.830 \\
\hline LCX & 63 & 37 & 0.893 \\
\hline $\mathrm{RCA}$ & 81 & 48 & 0.919 \\
\hline \multicolumn{4}{|c|}{ Degree of coronary stenosis } \\
\hline $90 \%<\mathrm{D}<100 \%$ & 54 & 20 & 0.038 \\
\hline $70 \%<\mathrm{D} \leq 90 \%$ & 125 & 62 & 0.086 \\
\hline $50 \% \leq \mathrm{D} \leq 70 \%$ & 67 & 66 & 0.001 \\
\hline
\end{tabular}

NSTEMI. The sensitivity of fQRS in 2 anterior ECG leads was the highest (80.9\%), but the specificity was only $68.4 \%$. The specificity of fQRS in 4 anterior ECG leads was the highest $(81.8 \%)$, but the sensitivity was only $62.7 \%$.

The sensitivity, specificity, and positive and negative predictive values of fQRS in ECG leads II, III, and aVF were $92.3 \%, 65.5 \%, 85.6$, and $79.2 \%$, respectively; the sensitivity, specificity, and positive and negative predictive values of fQRS in ECG leads I, aVL, and V6 were 89.4\%, $71.7 \%, 83.5$, and $80.9 \%$, respectively. Our results confirmed that the specificity of fQRS complexes in identifying lesions in the left circumflex and right coronary arteries was lower for the inferior and lateral leads than that for the limb leads (65.5\% versus $71.7 \%$ ); however, the former had higher sensitivity (92.3\% versus $89.4 \%)$ (Table 4).

\section{Comparison of the diagnostic accuracy between fQRS and} ischemic T-waves

The presence of fQRS for the diagnosis of left anterior artery (LAD) lesions was less sensitive $(58.0 \%$ versus $62.1 \%)$ but more specific $(75.00 \%$ versus $58.2 \%)$ compared with the presence of ischemic T-waves. The sensitivity and specificity of fQRS for the diagnosis of left circumflex artery (LCx) lesions were $89.4 \%$ and $71.7 \%$ compared with $53.4 \%$ and $70.6 \%$ for ischemic T-waves, respectively. For the diagnosis of right coronary artery (RCA) lesions, the presence of fQRS was more sensitive $(92.3 \%$ versus $66.2 \%)$ and less specific $(65.5 \%$ versus 66.3\%) than ischemic T-waves. We found that the total sensitivity and specificity of fQRS (77.1\% and $71.5 \%)$ were higher than those values for ischemic T-waves. 
Table 4 Electrocardiographic predictors of culprit lesions

\begin{tabular}{lllllll}
\hline & \multicolumn{7}{c}{ Predictors of LAD lesion } \\
\cline { 2 - 7 } & Sens & Spec & PPV & NPV & LR (+) & LR (-) \\
\hline fQRS in 2 anterior leads & $80.9 \%$ & $68.4 \%$ & $77.1 \%$ & $57.7 \%$ & 1.99 & 0.54 \\
fQRS in 3 anterior leads & $73.0 \%$ & $79.8 \%$ & $85.8 \%$ & $63.9 \%$ & 3.61 & 0.34 \\
fQRS in 4 anterior leads & $62.7 \%$ & $81.8 \%$ & $93.3 \%$ & $52.0 \%$ & 4.45 & 0.23 \\
fQRS in 5 anterior leads & $38.6 \%$ & $75.7 \%$ & $68.4 \%$ & $47.6 \%$ & 1.59 & 0.81 \\
& Predictors of RCA lesion & & \\
& Sens & Spec & PPV & NPV & LR (+) & LR (-) \\
fQRS in inferior leads & $92.3 \%$ & $65.5 \%$ & $85.6 \%$ & $79.2 \%$ & 2.68 & 0.12 \\
& Predictors of LCX lesion & & \\
& Sens & Spec & PPV & NPV & LR (+) & LR (-) \\
fQRS in I, aVL, and V6 leads & $89.4 \%$ & $71.7 \%$ & $83.5 \%$ & $80.9 \%$ & 3.16 & 0.15
\end{tabular}

$\mathrm{LAD}=$ left anterior descending artery; $\mathrm{RCA}=$ right coronary artery; $\mathrm{LCX}=$ left circumflex artery; Sens = sensitivity; Spec = specificity; PPV = positive predictive value; $N P V=$ negative predictive value; $L R=$ likelihood ratio.

Receiver operating characteristic (ROC) curves were used to evaluate the diagnostic accuracy of fQRS and ischemic T-waves for the diagnosis of culprit lesions in patients with NSTEMI. The areas under the ROC curves for fQRS and ischemic T-waves were 0.75 (95\% CI, $0.66-0.85$ ) and 0.54 (95\% CI, 0.41-0.64), respectively. Thus, the total diagnostic accuracy was significantly higher for fQRS than that for ischemic T-waves (Figure 1 and $2 ; p=0.03$ ).

\section{Discussion}

\section{Clinical risk factors}

In patients with NSTEMI, only a part of the artery is occluded. This implies that only a portion of

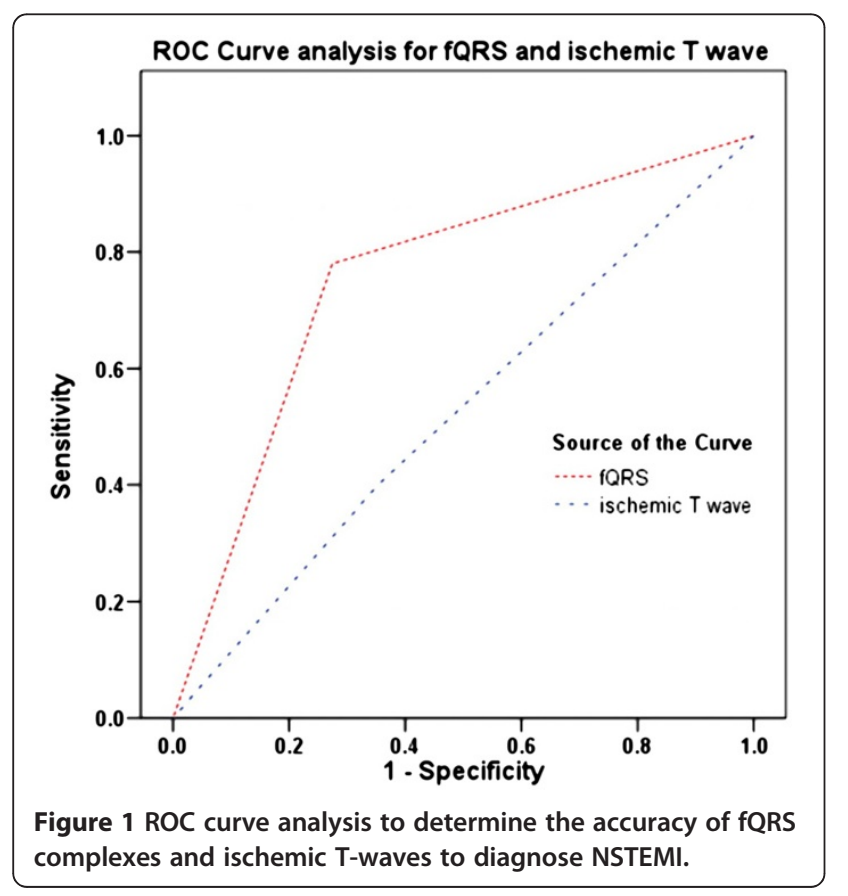

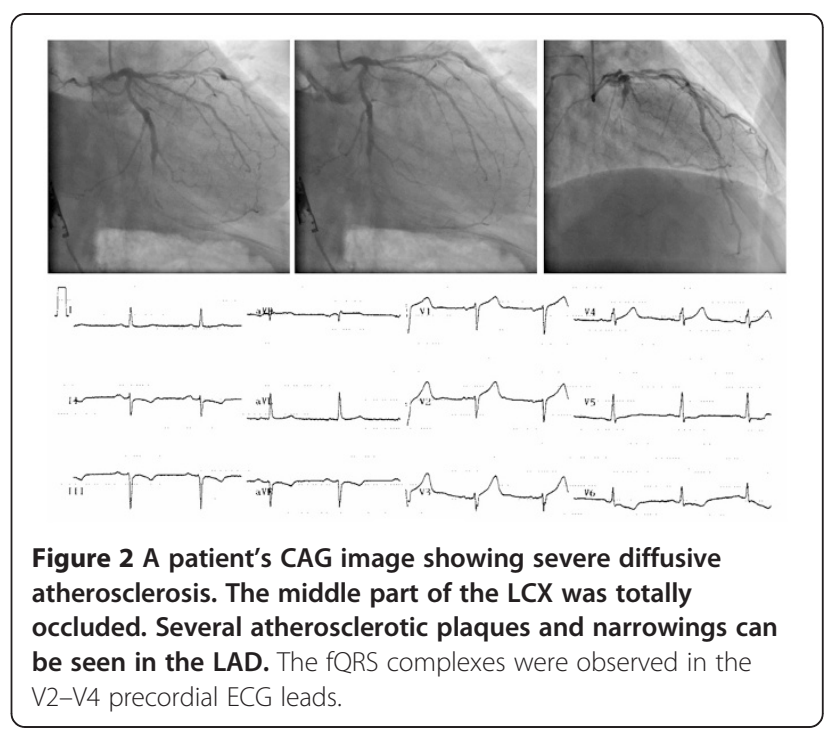

cardiomyocytes supplied by the occluded artery are necrotic. In the NSTEMI patients in the fQRS group, fQRS complexes were significantly more common in older patients ( $\geq 65$ years) and those with diabetes (OR, 2.04 [ $p=0.026]$ and OR, 2.05 [ $p=0.033]$, respectively). It has been established that the number of risk factors and complications are higher in elderly patients [8,9]. Moreover, a previous study has shown that diabetes can cause extensive vascular damage and increase the number of multivessel lesions [10]; this could explain the higher incidence of $\mathrm{fQRS}$ complexes in patients with diabetes.

\section{ECG analysis}

According to the standard distribution of the major coronary arteries in humans, fQRS complexes detected in 2 or more contiguous anterior leads (V1-V5) were assigned to the left anterior descending artery territory. The presence of fQRS complexes in the lateral leads (I, $\mathrm{aVL}$, and V6) were assigned to the left circumflex artery territory, and those in the inferior leads (II, III, and aVF) were assigned to the right coronary artery territory. We analyzed the frequency of fQRS in each lead and compared the frequencies with CAG findings. The frequency of fQRS was higher in the precordial leads than in other leads and showed higher specificity but lower sensitivity for predicting LAD lesions. The presence of fQRS complexes recorded in 4 precordial leads had the highest specificity for the identification of the culprit vessel (81.8\%) (Table 4). Although the specificity of the inferior and lateral lead recordings to identify LCX and RCA lesions was low (65.5\% versus $71.7 \%)$, the sensitivity was considerably higher (92.3\% versus $89.4 \%$ ).

ST-segment depression and/or T-wave inversion in the ECG usually reflects the abnormal repolarization of cardiac tissues caused by myocardial ischemia in patients 
with CAD. ST-segment elevation is an important ECG criterion for the diagnosis of STEMI, but the sensitivity and specificity of ST-segment depression and/or T-wave inversion for predicting the culprit lesion are not very high. Our results showed that the presence of fQRS complexes had better diagnostic accuracy than ischemic T-waves for the identification of culprit vessels (Figure 1; $p=0.03)$. These findings also suggest that $\mathrm{fQRS}$ could identify the correct culprit lesion in some patients without ischemic $\mathrm{T}$-wave changes.

Although the prognostic significance of fragmented QRS complexes in patients who underwent primary PCI was confirmed in previous studies, the diagnostic value of fQRS in patients who have a history of revascularization (PCI or CABG is uncertain $[2,4,11]$. We did not find a more frequent history of PCI or CABG in the fQRS group ( $p=0.525 ; p=0.917$ ). Furthermore, logistic regression analysis did not show that prior PCI or CABG were significantly associated with the presence of fQRS complexes $(p=0.973 ; p=0.834)$. Therefore, the diagnosis of culprit lesions in NESTEMI patients with a prior history of $\mathrm{CAD}$ and revasularization should be based on the combination of dynamic ECG changes including fQRS complexes and other clinical manifestations.

\section{Potential mechanisms}

The important of fQRS complexes was first suggested by Das in 2006. The exact mechanism of fragmentation of the QRS complex in the 12-lead surface ECG is still unclear. Previous studies have shown $[12,13]$ that the postinfarction scar tissue morphology correlated well with the patterns of the fQRS complex. The fQRS complexes have been implicated in the inhomogeneous activation of the ventricles due to myocardial scar and/or ischemia. These studies suggest that different fQRS patterns result from shifting of the QRS vector during depolarization of areas around $\mathrm{f}$ scar or ischemic myocardium, depending on the extent of injury and its location in the ventricles $[14,15]$.

\section{Conclusions}

Although there have been reports on the use of the fQRS complex as a marker of acute myocardial infarction and remote myocardial infarction scar, not much is known about the diagnostic significance of fQRS in patients with NSTEMI. We found that fQRS complexes occurred more frequently in elderly and diabetic patients with NSTEMI. The frequencies of fQRS complexes recorded in each ECG lead could identify the culprit vessel, and this was particularly useful when the LAD was the culprit vessel. In addition, the diagnostic accuracy of fQRS complexes was significantly higher than that of ischemic T-waves for the diagnosis of NSTEMI.

\section{Limitations}

There are several limitations of this study. The relatively small sample size limited the diagnostic value in clinical practice. Therefore, additional prospective data are needed in a larger study population to confirm our findings. Furthermore, the time that fQRS complexes developed in the ECG, and the exact mechanisms of fQRS are unknown at the present time. The utility of fQRS for identifying culprit lesions in NSTEMI patients with a history of PCI or CABG needs further exploration.

\section{Competing interest}

The authors declare no conflict of interest.

\section{Authors' contributions}

WL carried out the whole studies and participated in the patients' enrollment. YL and KT carried out the analysis and interpretation of ECGs. RG drafted the manuscript, participated in the design of the study and performed the statistical analysis. YX conceived of the study, participated in its design and coordination and helped to draft the manuscript. All authors read and approved the final manuscript.

Received: 11 October 2011 Accepted: 19 June 2012

Published: 19 June 2012

\section{References}

1. Das MK, Khan B, Jacob S, Kumar A, Mahenthiran J: Significance of a fragmented QRS complex versus a Q wave in patients with coronary artery disease. Circulation 2006, 113:2495-2501.

2. Das MK, Michael MA, Suradi H, Peng J, Sinha A, Shen C, Mahenthiran J, Kovacs RJ: Usefulness of fragmented QRS on a 12-lead electrocardiogram in acute coronary syndrome for predicting mortality. Am J Cardiol 2009, 104:1631-1637.

3. Kushner FG, Hand M, Smith SC Jr, King SB 3rd, Anderson JL, Antman EM, Bailey SR, Bates ER, Blankenship JC, Casey DE Jr, et al: 2009 focused updates: ACC/AHA guidelines for the management of patients with STelevation myocardial infarction (updating the 2004 guideline and 2007 focused update) and ACC/AHA/SCAI guidelines on percutaneous coronary intervention (updating the 2005 guideline and 2007 focused update) a report of the American College of Cardiology Foundation/ American Heart Association Task Force on Practice Guidelines. J Am Coll Cardiol 2009, 54:2205-2241

4. Das MK, Suradi H, Maskoun W, Michael MA, Shen C, Peng J, Dandamudi G, Mahenthiran J: Fragmented wide QRS on a 12-lead ECG: a sign of myocardial scar and poor prognosis. Circ Arrhythm Electrophysiol 2008, $1: 258-268$.

5. Haase J, Escaned J, van Swijndregt EM, Ozaki Y, Gronenschild E, Slager CJ, Serruys PW: Experimental validation of geometric and densitometric coronary measurements on the new generation Cardiovascular Angiography Analysis System (CAAS II). Cathet Cardiovasc Diagn 1993, 30:104-114.

6. Mueller HS DA, Greenberg MA, et al: The Thrombolysis in Myocardial Infarction (TIMI) trial. Phase I findings. TIMI Study Group. N Engl J Med 1985, 312:932-936.

7. Rentrop KP, Cohen M, Blanke H, Phillips RA: Changes in collateral channel filling immediately after controlled coronary artery occlusion by an angioplasty balloon in human subjects. J Am Coll Cardiol 1985, 5:587-592.

8. Brooks MM, Jones RH, Bach RG, Chaitman BR, Kern MJ, Orszulak TA, Follmann D, Sopko G, Blackstone EH, Califf RM: Predictors of mortality and mortality from cardiac causes in the bypass angioplasty revascularization investigation (BARI) randomized trial and registry. For the BARI Investigators. Circulation 2000, 101:2682-2689.

9. Best PJ, Lennon R, Ting HH, Bell MR, Rihal CS, Holmes DR, Berger PB: The impact of renal insufficiency on clinical outcomes in patients undergoing percutaneous coronary interventions. J Am Coll Cardiol 2002, 39:1113-1119. 
10. Donahoe SM, Stewart GC, McCabe CH, Mohanavelu S, Murphy SA, Cannon $\mathrm{CP}$, Antman EM: Diabetes and mortality following acute coronary syndromes. JAMA 2007, 298:765-775.

11. Ari H, Cetinkaya S, Ari S, Koca V, Bozat T: The prognostic significance of a fragmented QRS complex after primary percutaneous coronary intervention. Hear Vessel 2012, 27:20-28.

12. Flowers NC, Horan LG, Thomas JR, Tolleson WJ: The anatomic basis for high-frequency components in the electrocardiogram. Circulation 1969, 39:531-539.

13. Gardner PI, Ursell PC, Fenoglio JJ Jr, Wit AL: Electrophysiologic and anatomic basis for fractionated electrograms recorded from healed myocardial infarcts. Circulation 1985, 72:596-611.

14. el Sherif $\mathrm{N}$ : The rsR' pattern in left surface leads in ventricular aneurysm. Br Heart J 1970, 32:440-448.

15. Wiener I, Mindich B, Pitchon R: Fragmented endocardial electrical activity in patients with ventricular tachycardia: a new guide to surgical therapy. Am Heart J 1984, 107:86-90.

doi:10.1186/1471-2261-12-44

Cite this article as: Guo et al:: Significance of fragmented QRS complexes for identifying culprit lesions in patients with non-ST-elevation myocardial infarction: a single-center, retrospective analysis of 183 cases. BMC Cardiovascular Disorders 2012 12:44.

\section{Submit your next manuscript to BioMed Central and take full advantage of:}

- Convenient online submission

- Thorough peer review

- No space constraints or color figure charges

- Immediate publication on acceptance

- Inclusion in PubMed, CAS, Scopus and Google Scholar

- Research which is freely available for redistribution 